\title{
Erratum to: Calculations for deep inelastic scattering using fast interpolation grid techniques at NNLO in QCD and the extraction of $\alpha_{\mathrm{s}}$ from HERA data
}

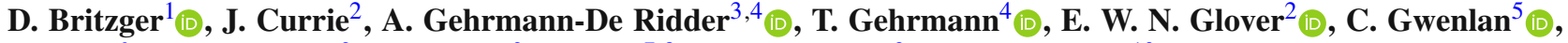

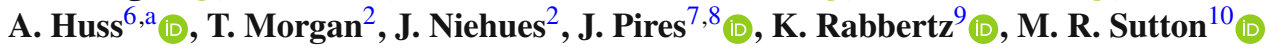 \\ ${ }^{1}$ Max-Planck-Institut für Physik, Föhringer Ring 6, 80805 Munich, Germany \\ ${ }^{2}$ Institute for Particle Physics Phenomenology, Durham University, Durham DH1 3LE, UK \\ ${ }^{3}$ Institute for Theoretical Physics, ETH, Wolfgang-Pauli-Strasse 27, 8093 Zurich, Switzerland \\ ${ }^{4}$ Physik-Institut, Universität Zürich, Winterthurerstrasse 190, 8057 Zurich, Switzerland \\ ${ }^{5}$ Department of Physics, The University of Oxford, Oxford OX1 3PU, UK \\ ${ }^{6}$ Theoretical Physics Department, CERN, 1211 Geneva 23, Switzerland \\ ${ }^{7}$ CFTP, Instituto Superior Técnico, Universidade de Lisboa, 1049-001 Lisbon, Portugal \\ ${ }^{8}$ LIP, Avenida Professor Gama Pinto 2, 1649-003 Lisbon, Portugal \\ ${ }^{9}$ Institut für Experimentelle Teilchenphysik (ETP), Karlsruhe Institute of Technology (KIT), Wolgang-Gaede-Str. 1, 76131 Karlsruhe, Germany \\ ${ }^{10}$ Department of Physics and Astronomy, The University of Sussex, Brighton BN1 9RH, UK
}

Received: 27 August 2021 / Accepted: 27 September 2021 / Published online: 29 October 2021

(C) The Author(s) 2021

\section{Erratum to: Eur. Phys. J. C (2019) 79:845 \\ https://doi.org/10.1140/epjc/s10052-019-7351-x}

The implementation of interpolation grid techniques at NNLO and their subsequent application to the extraction of the strong coupling constant $\alpha_{\text {s }}$ presented in Ref. [1] are based on the calculation in the NNLOJET framework [2-4]. An implementation error was found in this calculation [4] that altered the predicted cross sections for the DIS process at NNLO. While technical aspects and equations remain unchanged, reported numerical values and the extracted value of $\alpha_{\mathrm{s}}$ are affected. Updated figures, tables, and numbers quoted in the main text that have changed are provided. Numbering of sections and figures is as in Ref. [1].

\section{The APPLfast project}

Figure 2 compares the evaluation of the interpolation grids with the references obtained from NNLOJET for di-jet data at low $Q^{2}$ from $\mathrm{H} 1$ [5] and demonstrates an agreement better than the per-mille level across all bins.

The original article can be found online at https://doi.org/10.1140/ epjc/s10052-019-7351-x.

a e-mail: alexander.huss@cern.ch (corresponding author)
The different approaches pursued by APPLgrid and fastNLO in implementing the scale dependence are crosschecked against each other and found to be in mutual agreement. The resulting scale dependence is shown in Fig. 3 for two bins in inclusive jet $p_{\mathrm{T}}$; one from the $\mathrm{H} 1$ low $Q^{2}$ data [5] and one for the ZEUS high $Q^{2}$ data [6].

Figure 4 compares uncertainties that arise from the renormalisation and factorization scales (left) and the parton distribution functions (right) for the same $p_{\mathrm{T} \text {,jet }}$ distributions as considered in Fig. 3.

\section{Application: determination of the strong coupling constant}

An extraction of the strong coupling constant, $\alpha_{\mathrm{S}}\left(M_{\mathrm{Z}}\right)$, is performed using a fit of the NNLO QCD predictions from NNLOJET to the HERA inclusive jet cross-section data. Details on the fit procedure, the considered datasets [5-11], and internal checks are provided in the original publication [1].

Results for the values of $\alpha_{\mathrm{S}}\left(M_{\mathrm{Z}}\right)$ as obtained from the individual fits to the inclusive jet cross section data are collected in Table 1. The $\alpha_{\mathrm{S}}\left(M_{\mathrm{Z}}\right)$ values from the individual data sets are found to be mutually compatible within their respective errors. Figure 5 summarises the values for a visual comparison, and includes the world average [13], which is found to be consistent with the value extracted here. All the $\mathrm{H} 1$ and 

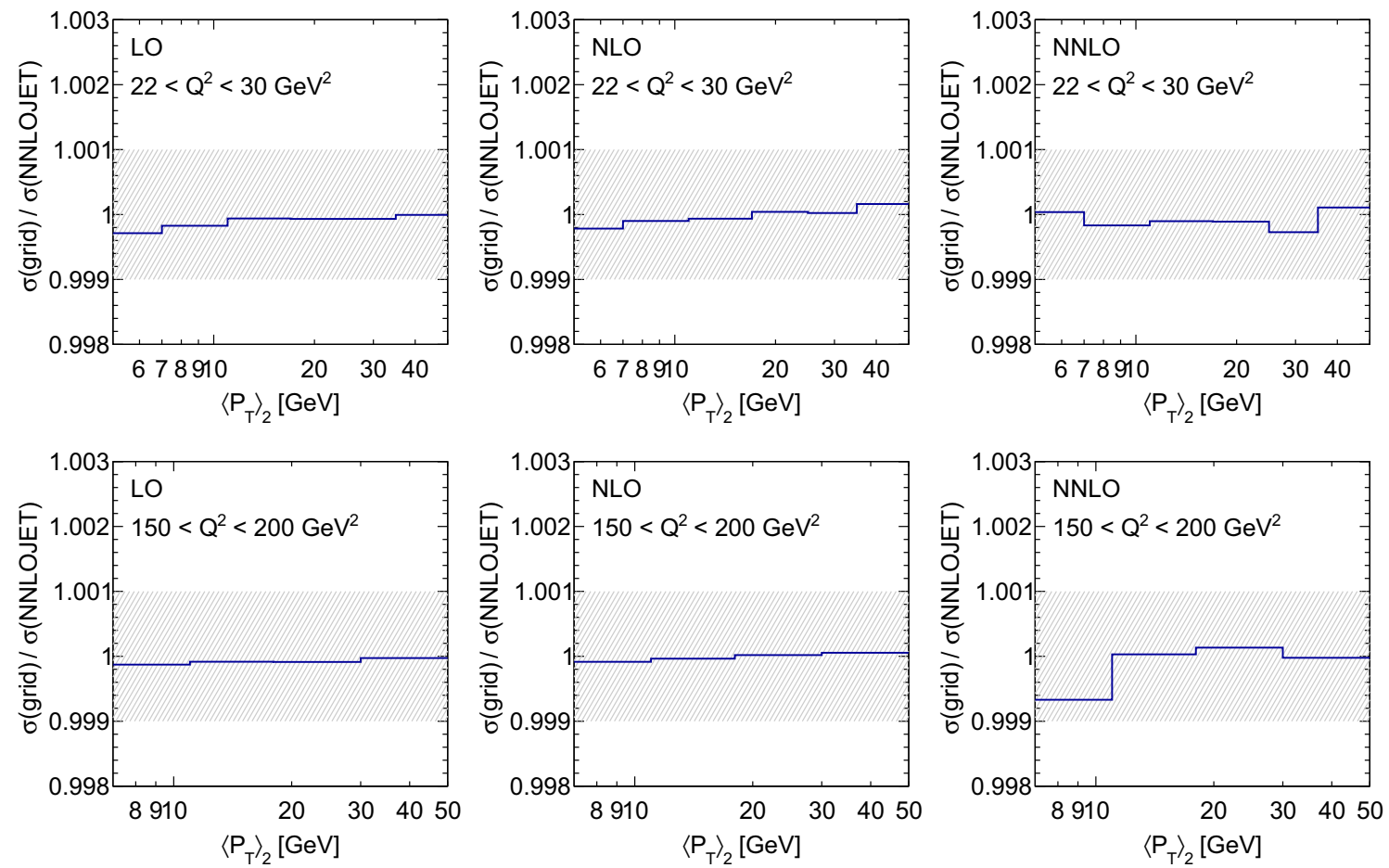

Fig. 2 Validation of the grid accuracy in di-jet production at low- $Q^{2}\left(22<Q^{2}<30 \mathrm{GeV}^{2}\right.$, top row) and high- $Q^{2}\left(150<Q^{2}<200 \mathrm{GeV}\right.$, bottom row). The shaded area indicates an agreement of $0.1 \%$

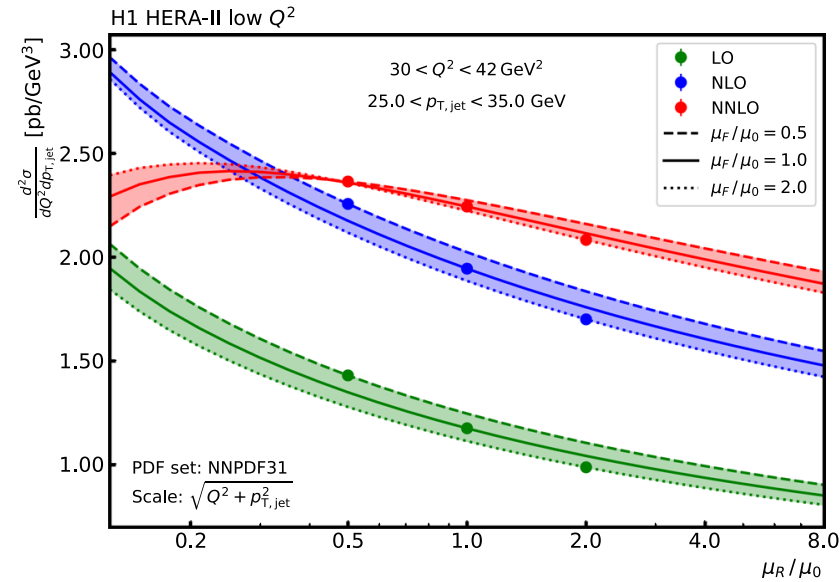

Fig. 3 The scale dependence for a single bin in jet $p_{\mathrm{T}}$ with $25<$ $p_{\mathrm{T}, \text { jet }}<35 \mathrm{GeV}$ for a range $30<Q^{2}<42 \mathrm{GeV}^{2}$ from $\mathrm{H} 1$ (left) and in jet $p_{\mathrm{T}}$ with $18<p_{\mathrm{T} \text {,jet }}<25 \mathrm{GeV}$ for a range $500<Q^{2}<1000 \mathrm{GeV}^{2}$ from ZEUS (right). The bands show

ZEUS inclusive jet cross section data are found to be in good agreement with the NNLO predictions, as indicated by the individual $\chi^{2} / n_{\text {dof }}$ values in Table 1 . From the fit to all HERA inclusive jet data a value of $\alpha_{\mathrm{s}}\left(M_{\mathrm{Z}}\right)=0.1171(9)_{\exp }(34)_{\text {th }}$ is obtained, where exp and th denote the experimental and theoretical uncertainties, respectively, and where the latter is obtained by combining individual theory uncertainties in quadrature. The fit yields $\chi^{2} / n_{\text {dof }}=170.7 / 193$, thus indi-

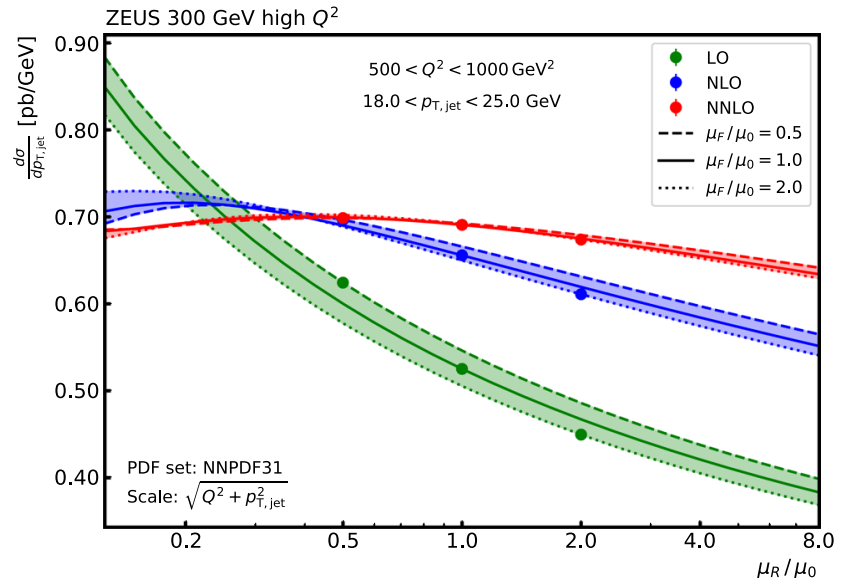

the result of varying the factorisation scale $\mu_{\mathrm{F}}$ by factors between 0.5 and 2.0 with respect to the nominal scale. At each order three points indicate the result of symmetric variations of $\mu_{\mathrm{R}}$ and $\mu_{\mathrm{F}}$

cating an excellent description of the data by the NNLO predictions. Furthermore, an overall high degree of consistency for all of the HERA inclusive jet cross section data is found.

The dominant uncertainty in the extraction of $\alpha_{\mathrm{S}}$ arises from the renormalisation scale dependence of the NNLO predictions. The fits are therefore repeated with a restricted data selection requiring $\tilde{\mu}>28 \mathrm{GeV}$, chosen to balance the experimental uncertainty from the measurements against the 

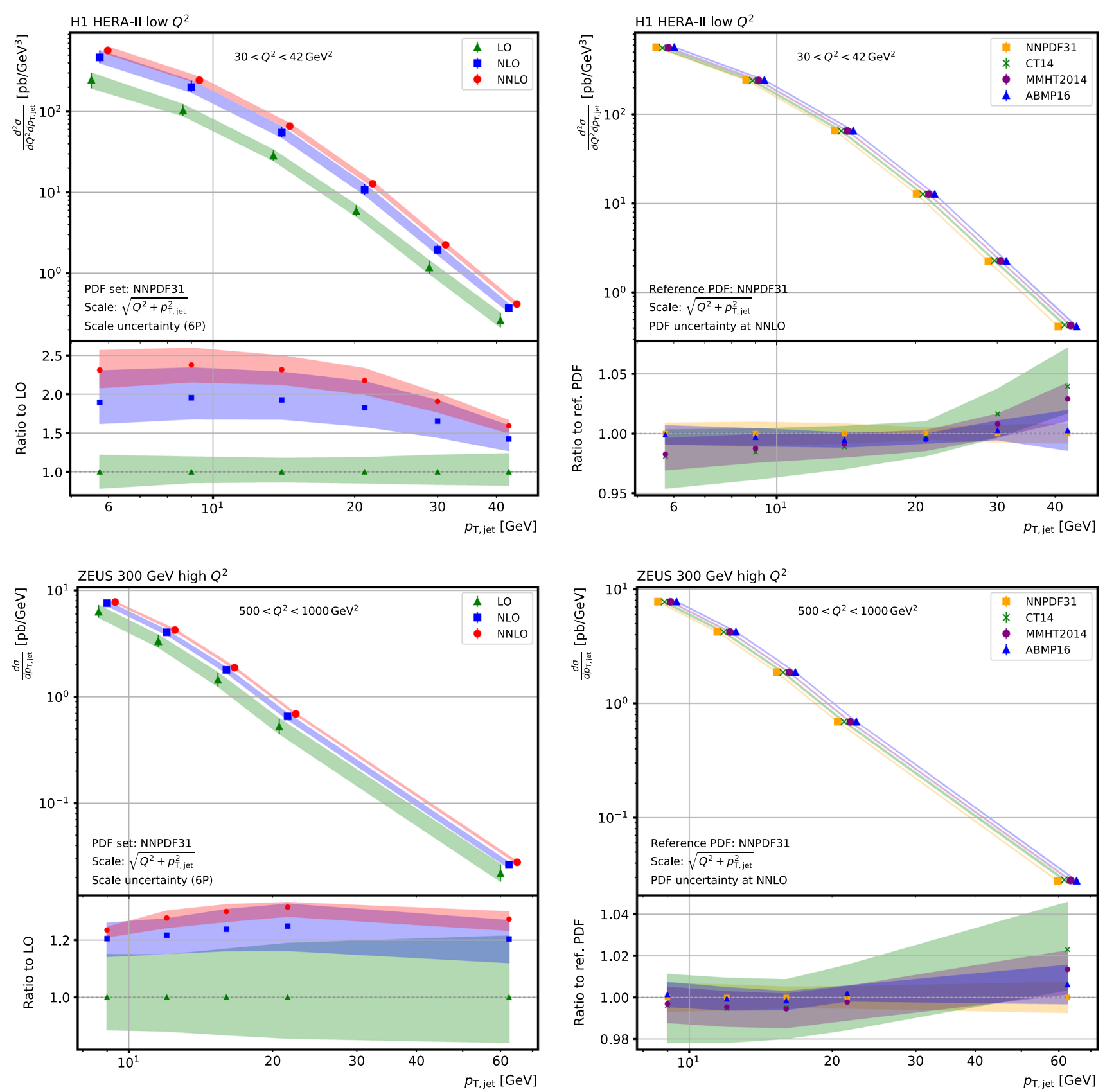

Fig. 4 Inclusive jet cross section as a function of the jet $p_{\mathrm{T}}$ for two ranges in $Q^{2}: 30<Q^{2}<42 \mathrm{GeV}^{2}$ for $\mathrm{H} 1$ data (upper row), and $500<Q^{2}<1000 \mathrm{GeV}^{2}$ for ZEUS data (lower row). On the left the LO, NLO, and NNLO predictions are shown using the NNPDF31 PDF set including their ratio to the LO in the respective lower panels. On the right the NNLO predictions are shown for the four PDF sets

NNPDF31, CT14, MMHT2014, and ABMP16 including their ratio to the NNPDF31 PDF prediction in the respective lower panels. The bands indicate the uncertainty derived from six variations of the $\mu_{\mathrm{R}}$ and $\mu_{\mathrm{F}}$ scale factors as described in the text (left), respectively the PDF uncertainty as prescribed in the respective publications. For better visibility the points in all upper panels are slightly shifted in $p_{\mathrm{T} \text {,jet }}$ 
Table 1 A summary of values of $\alpha_{\mathrm{S}}\left(M_{\mathrm{Z}}\right)$ from fits to HERA inclusive jet cross section measurements using NNLO predictions. The uncertainties denote the experimental (exp), hadronisation (had), PDF, PDF $\alpha_{s}$, PDFset and scale uncertainties as described in the text. The rightmost three columns denote the quadratic sum of the theoretical uncertainties (th), the total (tot) uncertainties and the value of $\chi^{2} / n_{\text {dof }}$ of the corresponding fit

\begin{tabular}{|c|c|c|c|c|c|}
\hline Data & $\tilde{\mu}_{\text {cut }}$ & $\alpha_{\mathrm{S}}\left(M_{\mathrm{Z}}\right)$ with uncertainties & th & tot & $\chi^{2} / n_{\text {dof }}$ \\
\hline \multicolumn{6}{|l|}{ H1 inclusive jets ${ }^{\dagger}$} \\
\hline $300 \mathrm{GeV}$ high- $Q^{2}$ & $2 m_{b}$ & $0.1253(33)_{\exp }(23)_{\text {had }}(5)_{\mathrm{PDF}}(3)_{\mathrm{PDF} \alpha_{\mathrm{s}}}(5)_{\mathrm{PDFset}}(28)_{\text {scale }}$ & $(37)_{\mathrm{th}}$ & $(49)_{\text {tot }}$ & $3.7 / 15$ \\
\hline HERA-I low- $Q^{2}$ & $2 m_{b}$ & $0.1113(18)_{\exp }(8)_{\text {had }}(5)_{\mathrm{PDF}}(5)_{\mathrm{PDF} \alpha_{\mathrm{s}}}(7)_{\mathrm{PDFset}}(33)_{\text {scale }}$ & $(36)_{\text {th }}$ & $(40)_{\text {tot }}$ & $14.6 / 22$ \\
\hline HERA-I high- $Q^{2}$ & $2 m_{b}$ & $0.1163(26)_{\exp }(9)_{\text {had }}(6)_{\mathrm{PDF}}(4)_{\mathrm{PDF} \alpha_{\mathrm{s}}}(3)_{\mathrm{PDFset}}(22)_{\text {scale }}$ & $(25)_{\mathrm{th}}$ & $(36)_{\text {tot }}$ & $13.2 / 23$ \\
\hline HERA-II low- $Q^{2}$ & $2 m_{b}$ & $0.1212(16)_{\exp }(12)_{\mathrm{had}}(4)_{\mathrm{PDF}}(4)_{\mathrm{PDF} \alpha_{\mathrm{s}}}(3)_{\mathrm{PDFset}}(38)_{\text {scale }}$ & $(40)_{\text {th }}$ & $(43)_{\text {tot }}$ & $28.2 / 40$ \\
\hline HERA-II high- $Q^{2}$ & $2 m_{b}$ & $0.1156(20)_{\exp }(10)_{\text {had }}(6)_{\mathrm{PDF}}(4)_{\mathrm{PDF} \alpha_{\mathrm{s}}}(2)_{\mathrm{PDFset}}(24)_{\text {scale }}$ & $(27)_{\text {th }}$ & $(34)_{\text {tot }}$ & $33.7 / 29$ \\
\hline \multicolumn{6}{|l|}{ ZEUS inclusive jets } \\
\hline $300 \mathrm{GeV}$ high- $Q^{2}$ & $2 m_{b}$ & $0.1240(30)_{\exp }(3)_{\text {had }}(5)_{\mathrm{PDF}}(1)_{\mathrm{PDF} \alpha_{\mathrm{s}}}(3)_{\mathrm{PDFset}}(17)_{\text {scale }}$ & $(18)_{\mathrm{th}}$ & $(35)_{\text {tot }}$ & $26.9 / 29$ \\
\hline HERA-I high- $Q^{2}$ & $2 m_{b}$ & $0.1211(29)_{\exp }(18)_{\text {had }}(5)_{\mathrm{PDF}}(1)_{\mathrm{PDF} \alpha_{\mathrm{s}}}(4)_{\mathrm{PDFset}}(14)_{\text {scale }}$ & $(24)_{\text {th }}$ & $(37)_{\text {tot }}$ & $18.1 / 29$ \\
\hline \multicolumn{6}{|l|}{ H1 inclusive jets ${ }^{\dagger}$} \\
\hline $\mathrm{H} 1$ inclusive jets & $2 m_{b}$ & $0.1157(10)_{\exp }(6)_{\mathrm{had}}(4)_{\mathrm{PDF}}(4)_{\mathrm{PDF} \alpha_{\mathrm{s}}}(2)_{\mathrm{PDFset}}(34)_{\text {scale }}$ & $(36)_{\mathrm{th}}$ & $(37)_{\text {tot }}$ & $118.1 / 133$ \\
\hline $\mathrm{H} 1$ inclusive jets & $28 \mathrm{GeV}$ & $0.1158(19)_{\exp }(9)_{\mathrm{had}}(2)_{\mathrm{PDF}}(2)_{\mathrm{PDF} \alpha_{\mathrm{s}}}(4)_{\mathrm{PDFset}}(21)_{\text {scale }}$ & $(23)_{\mathrm{th}}$ & $(30)_{\text {tot }}$ & $43.0 / 60$ \\
\hline \multicolumn{6}{|l|}{ ZEUS inclusive jets } \\
\hline ZEUS inclusive jets & $2 m_{b}$ & $0.1227(21)_{\exp }(9)_{\mathrm{had}}(6)_{\mathrm{PDF}}(1)_{\mathrm{PDF} \alpha_{\mathrm{s}}}(4)_{\mathrm{PDFset}}(16)_{\text {scale }}$ & $(19)_{\mathrm{th}}$ & $(28)_{\text {tot }}$ & $45.5 / 59$ \\
\hline ZEUS inclusive jets & $28 \mathrm{GeV}$ & $0.1208(25)_{\exp }(6)_{\text {had }}(4)_{\mathrm{PDF}}(2)_{\mathrm{PDF} \alpha_{\mathrm{s}}}(6)_{\mathrm{PDFset}}(15)_{\text {scale }}$ & $(18)_{\mathrm{th}}$ & $(31)_{\text {tot }}$ & $33.8 / 43$ \\
\hline \multicolumn{6}{|l|}{ HERA inclusive jets } \\
\hline HERA inclusive jets & $2 m_{b}$ & $0.1171(9)_{\exp }(5)_{\text {had }}(4)_{\mathrm{PDF}}(3)_{\mathrm{PDF} \alpha_{\mathrm{s}}}(2)_{\mathrm{PDFset}}(33)_{\text {scale }}$ & $(34)_{\text {th }}$ & $(35)_{\text {tot }}$ & $170.7 / 193$ \\
\hline HERA inclusive jets & $28 \mathrm{GeV}$ & $0.1178(15)_{\exp }(7)_{\text {had }}(2)_{\mathrm{PDF}}(2)_{\mathrm{PDF} \alpha_{\mathrm{s}}}(4)_{\mathrm{PDFset}}(19)_{\text {scale }}$ & $(21)_{\mathrm{th}}$ & $(26)_{\text {tot }}$ & $79.2 / 104$ \\
\hline
\end{tabular}

${ }^{\dagger}$ previously fit in Ref. [12]

scale dependence from the theory predictions and thus reduce the total uncertainty on the final extraction. This fit represents our main result and the value of $\alpha_{\mathrm{s}}\left(M_{\mathrm{Z}}\right)$ is determined to be

$\alpha_{\mathrm{s}}\left(M_{\mathrm{Z}}\right)=0.1178(15)_{\exp }(21)_{\mathrm{th}}$,

with the uncertainty decomposition given in Table 1 . The value is found to be consistent with the world average within uncertainties. The obtained uncertainties are competitive with other determinations from a single observable.

The running of $\alpha_{\mathrm{s}}\left(\mu_{\mathrm{R}}\right)$ can be inferred from separate fits to groups of data points that share a similar value of the renormalisation scale, as estimated by $\tilde{\mu}$. To this end, the $\alpha_{\mathrm{s}}\left(M_{\mathrm{Z}}\right)$ values are determined for each $\tilde{\mu}$ collection individually, and are summarised in Table 2 and shown in the bottom panel of Fig. 6. All values are mutually compatible and in good agreement with the world average, and no significant dependence on $\mu_{\mathrm{R}}$ is observed. The corresponding values for $\alpha_{\mathrm{s}}\left(\mu_{\mathrm{R}}\right)$, as determined using the QCD renormalisation group equation, are displayed in the top panel of Fig. 6, illustrating the running of the strong coupling. The dashed line corresponds to the prediction for the $\mu_{\mathrm{R}}$ dependence using the $\alpha_{\mathrm{s}}$ value of Eq. (19). The predicted running is in excellent agreement with the individual $\alpha_{\mathrm{S}}\left(\mu_{\mathrm{R}}\right)$ determinations, further reflecting the internal consistency of the study.

\section{Conclusions and outlook}

In this erratum, an implementation error in the underlying NNLO calculation is corrected [4]. Updated interpolation grids for inclusive jet cross sections at HERA were regenerated and provided on the ploughshare web site [14]. 
Table 2 Values of the strong coupling constant at the Z-boson mass, $\alpha_{\mathrm{s}}\left(M_{\mathrm{Z}}\right)$, obtained from fits to groups of data with comparable values of $\mu_{\mathrm{R}}$. The first (second) uncertainty of each point corresponds to the experimental (theory) uncertainties. The theory uncertainties include PDF related uncertainties and the dominating scale uncertainty

\begin{tabular}{llll}
\hline$\mu_{\mathrm{R}}$ & $\mathrm{H} 1$ & ZEUS \\
{$[\mathrm{GeV}]$} & $\alpha_{\mathrm{S}}\left(M_{\mathrm{Z}}\right)$ & $\alpha_{\mathrm{s}}\left(M_{\mathrm{Z}}\right)$ & $\begin{array}{l}\text { HERA } \\
\alpha_{\mathrm{s}}\left(M_{\mathrm{Z}}\right)\end{array}$ \\
\hline 7.4 & $0.1170(13)(41)$ & - & $0.1170(13)(41)$ \\
10.1 & $0.1161(17)(35)$ & - & $0.1161(17)(35)$ \\
13.3 & $0.1167(15)(41)$ & - & $0.1167(15)(41)$ \\
17.2 & $0.1161(15)(28)$ & $0.1220(28)(26)$ & $0.1176(13)(28)$ \\
20.1 & $0.1158(18)(28)$ & $0.1204(29)(22)$ & $0.1171(15)(26)$ \\
24.5 & $0.1184(16)(27)$ & $0.1221(27)(22)$ & $0.1195(14)(26)$ \\
29.3 & $0.1091(32)(31)$ & $0.1163(32)(20)$ & $0.1130(23)(24)$ \\
36.0 & $0.1164(27)(38)$ & $0.1221(28)(19)$ & $0.1196(19)(26)$ \\
49.0 & $0.1174(22)(17)$ & $0.1208(48)(27)$ & $0.1179(20)(18)$ \\
77.5 & $0.1082(51)(22)$ & $0.1266(44)(26)$ & $0.1191(34)(26)$ \\
\hline
\end{tabular}

$\alpha_{s}$ results from HERA inclusive jet data in NNLO

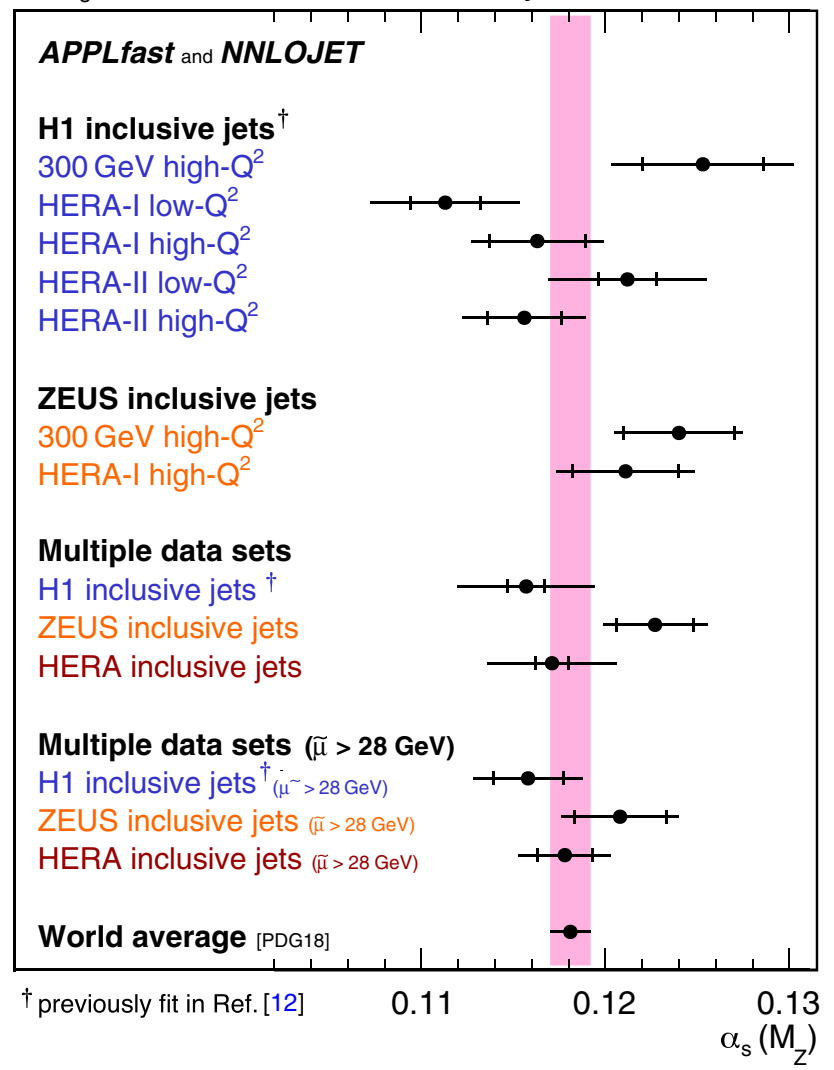

Fig. 5 Summary of $\alpha_{\mathrm{S}}\left(M_{\mathrm{Z}}\right)$ values in comparison with the world average value. The inner error bars indicate experimental uncertainties, and the full errors the total uncertainty, comprised of the experimental and theoretical uncertainties. The lower set of values represent fits to data restricted to $\tilde{\mu}>28 \mathrm{GeV}$

As an application of the grids, an extraction of the strong coupling constant $\alpha_{\mathrm{s}}$ has been performed, where inclusive jet cross section data from the H1 and ZEUS experiments at HERA are considered. Extracted values for $\alpha_{\mathrm{s}}$ are found to be consistently larger compared to the results presented in

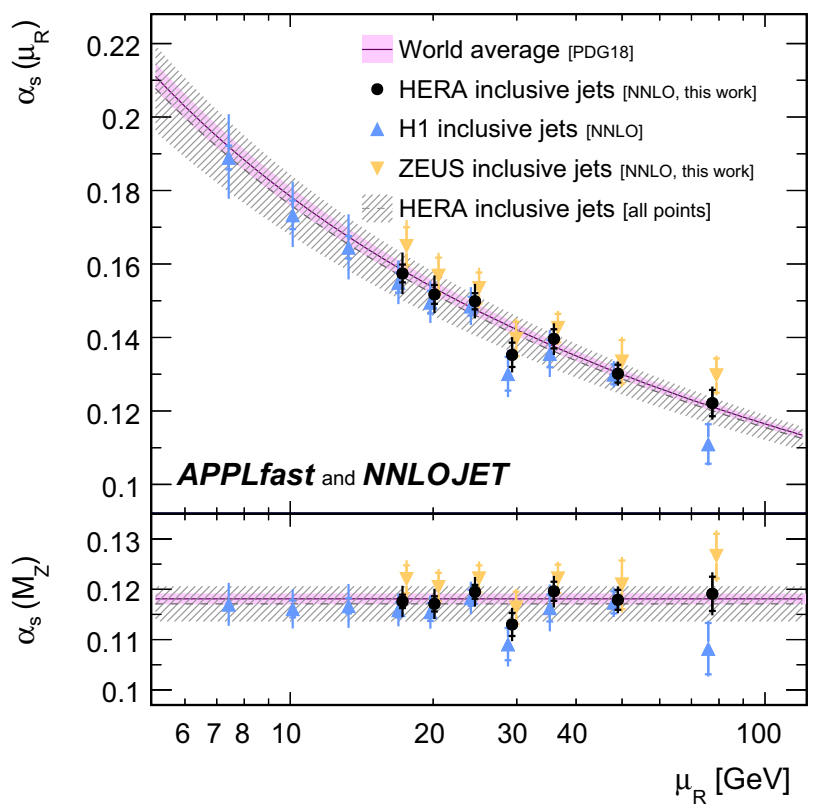

Fig. 6 Results for $\alpha_{\mathrm{S}}\left(M_{\mathrm{Z}}\right)$ (lower panel) and corresponding values for $\alpha_{\mathrm{S}}\left(\mu_{\mathrm{R}}\right)$ (upper panel) from fits to inclusive jet data points arranged in groups of similar $\mu_{\mathrm{R}}$. The upper panel is obtained by applying the expectation from the QCD renormalisation group equation, as it also enters the NNLO predictions. The inner error bars indicate experimental uncertainties, and the full error bars the total uncertainty. The upper triangles show results from H1 data, which were previously fit in Ref. [12] and are here partially updated with NNLO predictions with higher statistical accuracy. The lower triangles indicate the new results from ZEUS data. The full circles show the combined results from H1 and ZEUS data taken together and are labeled HERA inclusive jets. The shaded band indicates the world average value with its uncertainty, and the dashed line and hatched band indicate the result obtained from the fit to all inclusive jet data and its uncertainty

Ref. [1] and lie closer to the world average. The determination of $\alpha_{\mathrm{s}}\left(M_{\mathrm{Z}}\right)$ from $\mathrm{H} 1$ and ZEUS data taken together provides a best-fit value of $\alpha_{\mathrm{s}}\left(M_{\mathrm{Z}}\right)=0.1178(15)_{\exp }(21)_{\text {th }}$.

Data Availability Statement This manuscript has no associated data or the data will not be deposited. [Authors' comment: The datasets 
generated during and/or analysed during the current study are available on the ploughshare database, http://ploughshare.web.cern.ch.]

Open Access This article is licensed under a Creative Commons Attribution 4.0 International License, which permits use, sharing, adaptation, distribution and reproduction in any medium or format, as long as you give appropriate credit to the original author(s) and the source, provide a link to the Creative Commons licence, and indicate if changes were made. The images or other third party material in this article are included in the article's Creative Commons licence, unless indicated otherwise in a credit line to the material. If material is not included in the article's Creative Commons licence and your intended use is not permitted by statutory regulation or exceeds the permitted use, you will need to obtain permission directly from the copyright holder. To view a copy of this licence, visit http://creativecomm ons.org/licenses/by/4.0/.

Funded by SCOAP ${ }^{3}$.

\section{References}

1. D. Britzger et al., Eur. Phys. J. C 79, 845 (2019). https://doi.org/ 10.1140/epjc/s10052-019-7351-x. arXiv:1906.05303

2. A. Gehrmann-De Ridder, T. Gehrmann, E.W.N. Glover, A. Huss, T.A. Morgan, JHEP 07, 133 (2016). https://doi.org/10.1007/ JHEP07(2016)133. arXiv:1605.04295

3. J. Currie, T. Gehrmann, J. Niehues, Phys. Rev. Lett. 117, 042001 (2016). https://doi.org/10.1103/PhysRevLett.117.042001. arXiv:1606.03991

4. J. Currie, T. Gehrmann, A. Huss, J. Niehues, JHEP 07, 018 (2017). https://doi.org/10.1007/JHEP07(2017)018. arXiv:1703.05977. [Erratum: JHEP 2012, 042 (2020)]
5. H1 Collaboration, V. Andreev et al., Eur. Phys. J. C 77, 215 (2017). https://doi.org/10.1140/epjc/s10052-017-4717-9. arXiv:1611.03421. [Erratum: Eur. Phys. J. C 81, 739 (2021)]

6. ZEUS Collaboration, S. Chekanov et al., Nucl. Phys. B 765, 1 (2007). https://doi.org/10.1016/j.nuclphysb.2006.09.018. arXiv:hep-ex/0608048

7. H1 Collaboration, C. Adloff et al., Eur. Phys. J. C 19, 289 (2001). https://doi.org/10.1007/s100520100621. arXiv:hep-ex/0010054

8. H1 Collaboration, F.D. Aaron et al., Eur. Phys. J. C 67, 1 (2010). https://doi.org/10.1140/epjc/s10052-010-1282-x. arXiv:0911.5678

9. H1 Collaboration, A. Aktas et al., Phys. Lett. B 653, 134 (2007). https://doi.org/10.1016/j.physletb.2007.07.050. arXiv:0706.3722

10. H1 Collaboration, V. Andreev et al., Eur. Phys. J. C 75, 65 (2015). https://doi.org/10.1140/epjc/s10052-014-3223-6. arXiv: 1406.4709

11. ZEUS Collaboration, S. Chekanov et al., Phys. Lett. B 547, 164 (2002). https://doi.org/10.1016/S0370-2693(02)02763-6. arXiv:hep-ex/0208037

12. H1 Collaboration, V. Andreev et al., Eur. Phys. J. C 77, 791 (2017). https://doi.org/10.1140/epjc/s10052-017-5314-7. arXiv:1709.07251. [Erratum: Eur. Phys. J. C 81, 738 (2021)]

13. Particle Data Group Collaboration, M. Tanabashi et al., Phys. Rev. D 98, 030001 (2018). https://doi.org/10.1103/PhysRevD.98. 030001

14. ploughshare. http://ploughshare.web.cern.ch 